\title{
Theatre Performance Dashboard: Development and Challenges
}

\author{
Ahmad ABDEL-HAFEZ ${ }^{\mathrm{a}, 1}$, Michelle WINNING ${ }^{\mathrm{a}}$ and Michael GILL ${ }^{\mathrm{a}}$ \\ ${ }^{a}$ Metro South Health, Queensland, Australia
}

\begin{abstract}
Manual theatre performance measurement is resource yearning and inaccurate. To automate the process, we built a dashboard which provides interactive visualisation of key performance metrics related to operating theatres. The aim is to assist in the efficient management of surgical services and provide visibility on metrics trending over time for health service facilities.
\end{abstract}

Keywords. Dashboard, data visualisation, decision support, clinical informatics

\section{Introduction}

Surgical services are one of the most critical and expensive resources in any hospital. The introduction of the integrated electronic medical record (ieMR) has provided a new and rich repository of clinically inputted data. This ieMR data has now been used to build analytical tools that provide hospital executives and health professionals with insights into theatre-related performance metrics to assist in the efficient management of surgical services, such as operating rooms usage and utilisation [1].

Previously, data collection and theatre performance calculations required significant resourcing to manually extract and manipulate available information. Limitations of data visibility and integration resulted in reports that health professionals had low confidence in. This addresses the need for an interactive tool to calculate and display accurate information and trending for the theatre performance.

\section{Methods}

We built a dashboard (Figure 1.) to report key performance metrics, and provide trending data, with the ability to drill down and interrogate performance for a specific location or specialty. The data sources for this project were collected from two systems that are used together for theatre management; Enterprise Scheduling Management system (ESM) and SurgiNet.

After several meetings with surgical department teams we collated a requirements document describing key performance metrics. These metrics aligned to the operating theatre efficiency document published by Queensland Health [2]. Examples of the dashboard indicators are total patients treated, late starts, finishing on time - overruns and underruns, elective cancellations day of surgery, average changeover time, and

\footnotetext{
${ }^{1}$ Corresponding Author, Ahmad Abdel-Hafez; E-mail: ahmad.abdel-hafez@health.qld.gov.au.
} 
elective theatre utilisation. In the absence of existing fit for purpose SurgiNet reporting, the team had to embark on a large piece of work around data discovery and exploration. Significant clinical validation was completed by the local experts to ensure the outputs were in alignment with the business requirements.

During this project we overcame many challenges due to the complex nature of SurgiNet and ESM modules. Detailed mapping of local workflows was required to garner an intricate understanding of the booking and cancellation process within the system and an appreciation of the interaction between the two modules. One example is the utilisation of an elective session metric, where the elective sessions are created on ESM system and surgery data entered on SurgiNet system. The second large undertaking was the dashboard validation process. This process was conducted by a team of clinicians (experts in using ESM and SurgiNet systems). The coordination between the teams to validate one metric that used data from both systems was not a simple task. This was addressed by splitting metrics into several granular elements which were validated separately. When all the elements of the specific metric were validated successfully, the final value was confirmed to be accurate.

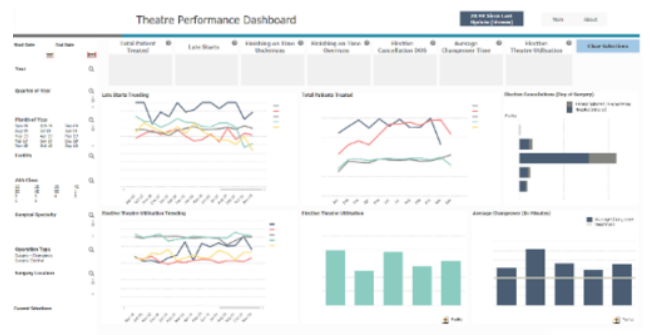

Figure 1. Theatre performance dashboard.

\section{Results}

Feedback from clinicians regarding this product was extraordinary, and we received several requests to expand this work to include other health services. In future, we will conduct a staff survey to measure the impact of using the dashboard on the theatres' KPIs.

\section{Conclusions}

We have built a dashboard to reflect key performance metrics at a tertiary level hospital. During this process we faced many challenges; primarily understanding complex business processes, validation, and multidisciplinary team management. The dashboard is expected to influence workflows and process change in the future. 


\section{References}

[1] Kargar ZS, Khanna S, Sattar A. Using prediction to improve elective surgery scheduling. The Australasian medical journal 6(5), 287, 2013.

[2] Queensland Health Guidelines. Operating Theatre Efficiency Guideline, State of Queensland (Queensland Health), Brisbane QLD 4000, Australia, 2017. 